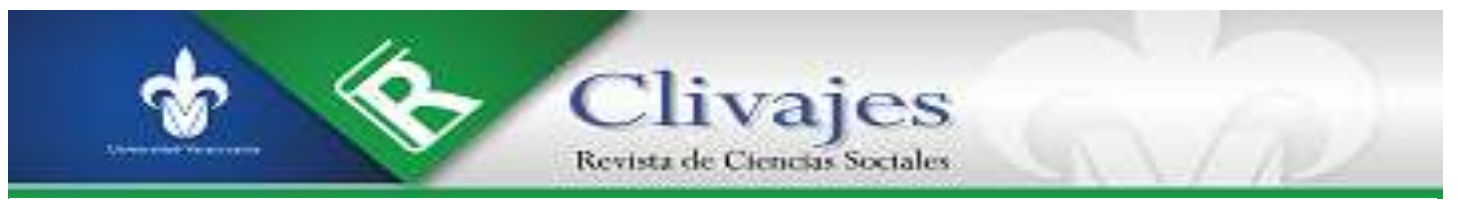

María Elena Meza de Luna, mezamariel@gmail.com

Lorena Erika Osorio Franco, lorenaosorio030@hotmail.com

Amanda Hernández Pérez, hernandez.perez.am@gmail.com

Facultad de Ciencias Políticas y Sociales, Universidad Autónoma de Querétaro

PERCEPCIÓN DE ADOLESCENTES SOBRE LAS PRÁCTICAS DE CRIANZA DE SUS PADRES Y MADRES:

COMPARATIVO DE GÉNERO EN CONTEXTOS RURAL Y URBANO

DOI: https://doi.org/10.25009/clivajes-rcs.v0i14.2672

Clivajes. Revista de Ciencias Sociales. Año VII, número 14, julio-diciembre 2020, pp. 183-205.

https:// clivajes.uv.mx/index.php/Clivajes/article/view/2672/4481

Instituto de Investigaciones Histórico-Sociales, Universidad Veracruzana

Clivajes. Revista de Ciencias Sociales/ISSN: 2395-9495/IIH-S, UV/Xalapa, Veracruz, México

Recibido: $18 / 02 / 2020$

Aceptado: 20/02/2020

Dictaminado: 05/10/2020

Clivajes. Revista de Ciencias Sociales (ISSN: 2395-9495), Año VII, Núm. 14, julio-diciembre, 2020

Instituto de Investigaciones Histórico-Sociales, Universidad Veracruzana, México 


\title{
PERCEPCIÓN DE ADOLESCENTES SOBRE LAS PRÁCTICAS DE CRIANZA DE SUS PADRES Y MADRES: COMPARATIVO DE GÉNERO EN CONTEXTOS RURAL Y URBANO
}

\author{
María Elena Meza de Luna* \\ Lorena Erika Osorio Franco** \\ Amanda Hernández Pérez ${ }^{* * *}$
}

\begin{abstract}
Resumen
Las relaciones en familia son fundamentales para la vida de las personas, en especial para las/los adolescentes en cuyo futuro tienen un fuerte impacto, de ahí la importancia de dimensionarlas, a fin de orientar el desarrollo de políticas públicas para la familia. En este artículo damos cuenta de una investigación cuyo objetivo fue evaluar y comparar la percepción de las/los adolescentes con respecto a las prácticas de crianza de sus madres/padres, en función del género de sus hijos/hijas y de su residencia en contextos rural o urbano. Se trata de una investigación cuantitativa y transversal, con representatividad municipal en San Juan del Río, Querétaro, donde se encontró un alto nivel de prácticas permisivas de madres/padres, además de que existe mayor riesgo para los hombres del área urbana, que para los de la rural, de experimentar lejanía emocional de sus padres. Se concluye que, aunque las/los adolescentes tienden a evaluar de manera similar la ejecución de las prácticas de crianza, aún refieren reminiscencias de trato diferenciado por género y contexto.
\end{abstract}

Palabras clave: Prácticas de crianza, Adolescentes, Género, Contextos rural y urbano

PERCEPTION OF ADOLESCENTS ABOUT THE PARENTING PRACTICES OF THEIR FATHERS AND MOTHERS. COMPARATIVE GENDER IN RURAL AND URBAN CONTEXTS

\section{Summary}

Family relationships are essential for people's lives, especially for adolescents in whose future they have a strong impact, hence the importance of dimensioning them, in order to guide the development of public policies for the family. In this article, an investigation whose objective was to evaluate and compare the perception of adolescents regarding the parenting practices of their mothers / fathers, based on the gender of their children and their residence in rural or urban contexts, was reported. It is a quantitative and cross-sectional investigation, with municipal representation in San Juan del Río, Querétaro, where a high level of permissive practices of mothers / fathers was found, in addition to the fact that there is a greater risk for men in the urban area, than for those of the rural area, to experience emotional distance from their parents. It is concluded that, although adolescents tend to evaluate the performance of parenting practices in a similar way, they still refer reminiscences of differentiated treatment by gender and context.

Keywords: Parenting practices, Adolescents, Gender, Rural and urban contexts

PERCEPTION D’ ADOLESCENTS SUR LES PRATIQUES D'ÉDUCATION DE LEURS PÈRES ET MÈRES. COMPARATIVE DE GENRE EN CONTEXTES RURAL ET URBAIN

\section{Résumé}

Les relations en famille sont fondamentales pour la vie des personnes, spécialement pour les adolescents sur qui elles ont un grand impact, voilà l'importance de les dimensionner à fin d'orienter le développement de politiques publiques pour la

${ }^{*}$ Profesora-investigadora. Facultad de Ciencias Políticas y Sociales, Universidad Autónoma de Querétaro, México. Contacto: mezamariel@gmail.com.

Profesora-investigadora. Facultad de Ciencias Políticas y Sociales, Universidad Autónoma de Querétaro, México. Contacto: lorenaosorio030@hotmail.com.

*** Profesora-investigadora. Facultad de Ciencias Políticas y Sociales, Universidad Autónoma de Querétaro, México. Contacto: hernandez.perez.am@gmail.com.

Clivajes. Revista de Ciencias Sociales (ISSN: 2395-9495), Año VII, Núm. 14, julio-diciembre, 2020 Instituto de Investigaciones Histórico-Sociales, Universidad Veracruzana, México 
famille. Dans cet article nous rendons compte d'une recherche dont l'objectif a été celui d'évaluer et de comparer la perception des adolescents envers les pratiques d'éducation de leurs mères et pères en fonction du genre de leurs fils/filles et de leur résidence en contextes rural et urbain. Il s'agit d'une recherche quantitative et transversale avec représentativité municipale à San Juan del Río, Querétaro, où on a trouvé un grand niveau de pratiques permissives des mères/pères, en plus de l'existence d'un plus grand risque pour les hommes de l'are urbain que pour ceux de l'are rural d'expérimenter l'éloignement émotionnel de leurs parents. On conclue que même les adolescents ont une tendance à évaluer de manière similaire la mise en ouvre des pratiques d'éducation, ils font référence à des réminiscences d'un traitement différencié par genre et contexte.

Mots clés : Pratiques d'éducation, Adolescents, Genre, Contextes rural et urbain

\section{INTRODUCCIÓN}

En el ámbito de la familia se da una de las relaciones intergeneracionales primarias, donde padres y madres establecen prácticas, valores e impresiones profundas y duraderas en sus hijos/as (Segrin \& Flora, 2011). Las familias tejen vínculos de largo plazo que juegan un rol importante en el proceso de socialización; esto es, en los intercambios cotidianos entre sus integrantes, quienes, con base en la repetición acumulada, van modelando el comportamiento social de los/as hijos/as (Snyder, 2016). Una adecuada relación incluye interacciones simétricas; un diálogo que genera confianza mutua y promueve el desarrollo de las personas (Giddens \& Sutton, 2014).

Al proceso de cuidado, apoyo, socialización, atención y educación que dirigen las personas adultas a los/as menores de edad, se le conoce como crianza, que se ha definido “como los conocimientos, actitudes y creencias que los padres asumen en relación con la salud, la nutrición, la importancia de los ambientes físico y social, y las oportunidades de estimulación y aprendizaje de los hijos" (Eraso, Bravo \& Delgado, 2006, p. 1). Aunque la crianza la puede ejercer cualquier persona (Consejo de Europa, Comité de Ministros, 2006), este artículo se circunscribe a la que ejercen padres y madres.

La crianza está atravesada por 1 . normativas socioculturales; 2. creencias propias de lo que corresponde ser y hacer como padre/madre; y, finalmente, 3. prácticas de madres/padres, es decir, acciones concretas que padres/madres dirigen hacia sus hijos/as en este proceso de crianza, y que se entretejen con las normas y creencias (Izzedin \& Pachajoa, 2009).

El ejercicio de la crianza puede asociarse a patrones que implican cierta regularidad en el comportamiento de padres/madres, a lo que se conoce como estilos de crianza, que se caracterizan caracterizados por dos componentes primarios: 1) el grado de control y 2) el grado de apoyo. Su combinación deriva en cuatro estilos de crianza, a los que Baumrind llama: a) autoritario, b) democrático o autoritativo, c) negligente, y d) permisivo (Segrin 
\& Flora, 2011). El más deseable para el sano desarrollo de niños/as y adolescentes es el democrático, que en tiempos recientes se ha asociado con la crianza positiva, basada en una filosofía y práctica alejada de la violencia, al tiempo que ofrece reconocimiento, orientación, establecimiento de rutinas, acompañamiento, afecto, apoyo, comunicación asertiva, límites y normas claras (Consejo de Europa, Comité de Ministros, 2006).

Los nexos familiares tienen influencia en la personalidad, en las elecciones futuras y en el desarrollo personal y profesional de los/as adolescentes (Niedorys, Mikos, Rząca \& Kocka, 2018). Así, los estilos de crianza a los que se les expone tienen un efecto crucial en su vida; por ejemplo, las prácticas negligentes - con bajo control e insuficiente apoyo emocional (e.g., con escaso diálogo o respeto)- muestran una asociación prospectiva, con inclinación al despliegue de conductas antisociales e incremento de rasgos de insensibilidad, manifiestas en falta de empatía y poca respuesta emocional en cada estadio del desarrollo de los/as hijos/as (Pardini \& Loeber, 2008; Waller, Gardner \& Hyde, 2013). La negligencia en las prácticas de padres/madres es, también, un factor de riesgo para el consumo de sustancias psicoactivas (Valente, Cogo \& Zila, 2018) y la adicción al uso de la Internet (Niedorys et al., 2018).

Por otro lado, el estilo de crianza permisivo - con bajo nivel de control y alto apoyo emocional- es un factor de riesgo para que los/as hijos/as sean víctimas del bullying (Charalampous, Demetriou, Tricha \& Loannou, 2018), mientras que el autoritarismo en la crianza - con alto control y bajo apoyo emocional- está asociado con la deserción escolar (Snyder, 2016) y, en el contexto mexicano, se ha relacionado con hiperactividad, problemas de conducta, problemas con compañeros y síntomas emocionales (Méndez Sánchez, Peñaloza Gómez, García Méndez, Jaenes Sánchez \& Velázquez Jurado, 2019), consumo de alcohol (Cruz, Flores \& Jiménez, 2019), ansiedad (Ruvalcaba Romero, Gallegos Guajardo, Caballo \& Villegas Guinea, 2016) y sintomatología depresiva (Andrade, Betancourt, Vallejo, Segura \& Rojas, 2012).

Por lo contrario, la crianza positiva, que se concreta con buena comunicación con los/as adolescentes y supervisión cercana, es un factor protector contra el consumo de alcohol (Oman et al., 2007) y mariguana (Betancourt et al., 2018); además, está asociada con el desarrollo del liderazgo en la adultez, con alejarse de apegos emocionales nocivos (Eldad \& Benatov, 2008); es un potenciador del desarrollo cognitivo (Vargas Rubilar \& Arán Filippetti, 2014) y de la autoestima (Estrada \& Rodríguez, 2012), y predictor de la autoeficacia para sobrellevar las desventajas de vivir en contextos adversos (e. g., barrios violentos) (Ardelt \& Eccles, 2001). En suma, la calidad de las relaciones entre 
padres/madres e hijos/as, circunscrita en la crianza, es crítica para el desarrollo de los/as adolescentes (Dangi \& Witt, 2016).

Asimismo, hay que considerar que los contextos condicionan el desarrollo de las familias (Zhang \& Fuligni, 2006). En el ejercicio de las prácticas de crianza influyen cuestiones como las condiciones del lugar donde se asienta la familia (e. g., por la seguridad e infraestructura del vecindario) y la cultura propia de la comunidad, en la que se entretejen, por ejemplo, las creencias sobre la legitimidad de la autoridad que tienen madres/padres y de cómo deben ejercerla (Smetana, 2017). Así, sabiendo que vivir en una zona urbana, o una rural, puede afectar de modo distinto el cómo las personas enfrentan el estrés y los problemas de la vida (Voth, 2001), es pertinente evaluar las posibles diferencias entre las prácticas de crianza en el medio rural y urbano.

En la escasa literatura internacional que compara dichas prácticas en el contexto rural-urbano, hay indicios de que cuando se alejan de rasgos positivos (i.e., siendo permisivas, negligentes o violentas) estas diferencias están asociadas con la ansiedad en adolescentes, y afectan más a los adolescentes de contextos urbanos (ACU) que a los de entornos rurales (Yu et al., 2010). Al estudiar a adolescentes chinos, Zhang y Fuligni (2006) encontraron que los ACU recibían un menor número de reglas que los adolescentes en el contexto rural (ACR). En otros estudios se ha encontrado también que madres colombianas en estratos urbanos tienden a usar más violencia verbal y física con sus hijos/as, que las de medio rural, y se estima que puede deberse a que son más proclives a situaciones de estrés tanto sociales y laborales como familiares (Rodríguez Villamizar \& Amaya Castellanos, 2019). Sin embargo, en México, a pesar de la abundante producción científica sobre las prácticas de crianza, existe un vacío en estudios que aborden este análisis comparativo rural-urbano, de ahí la importancia de considerar la voz de la adolescencia en estos asuntos de su vida diaria (Casais, Flores \& Domínguez, 2017).

Asimismo, cabe considerar que el sistema sexo-género se interioriza en primera instancia a través de los procesos de socialización dentro de la familia, siendo madres y padres los principales agentes socializadores. En ese sentido, los constructos de género atraviesan los estilos de crianza (Cantón, Ramírez \& Cantón, 2014), pues la socialización puede llegar a ser diferenciada entre hijas e hijos, y con ello no sólo se perpetúa las desigualdades de género, sino que se les encuentra normalizadas (Leaper \& Farkas, 2015); por ejemplo, sobre los hijos, por ser hombres, se llega a considerar que están más seguros y que requieren menos cuidado que las hijas, a quienes se supone más frágiles y vulnerables (Rengifo \& Martínez, 2014). También, se ha reportado que a los adolescentes varones se les somete a mayores actos de disciplina que a las mujeres (Calvete, Guadix \& 
Orue, 2010) e incluso que padres con actitudes igualitarias pueden actuar, sin embargo, de manera diferenciada con sus hijas e hijos (Leaper \& Farkas, 2015). No obstante, las investigaciones sobre las prácticas de crianza según el género no son concluyentes; por ello, es importante seguir explorando el tema.

Considerando lo antedicho, el objetivo de la investigación fue evaluar y comparar la percepción de los/as adolescentes con respecto al ejercicio de prácticas de crianza de sus madres/padres en función de su género y su residencia en el contexto rural o urbano.

\section{MATERIALES Y MÉTODOS}

Se realizó una investigación cuantitativa de corte retrospectivo, de tipo transversal y con diseño no experimental. Con base en los antecedentes, se sabe que el contexto donde se asienta la familia influye en el ejercicio de la crianza (Smetana, 2017), en parte porque existen pautas culturales, cogniciones y recursos diferentes. Por tanto, dado que hay evidencia de que en el contexto rural y urbano las sociedades divergen para encarar los propios problemas de la vida y varían los recursos disponibles (Voth, 2001), es pertinente realizar un estudio comparativo urbano-rural.

La investigación se realizó en San Juan del Río, un municipio bastión para el establecimiento industrial y empresarial en el estado de Querétaro. El Instituto Nacional de Estadística, Geografía e Informática (INEGI, 2015) reportó que tenía 268408 habitantes, con una población, de 25285 jóvenes de entre 15 y 19 años. En cuanto a la población estudiantil en ese municipio, encontramos que en el ciclo escolar 2016-2017, hubo 11018 alumnos de bachillerato. Según el Plan de Desarrollo Municipal de San Juan del Río, a consecuencia de la migración ha habido un incremento poblacional del $4.9 \%$ (con $6.1 \%$ de inmigrantes y $1.2 \%$ de emigrantes), lo cual lo ubica como el tercer municipio del estado de Querétaro - después de Corregidora y El Marqués- en atracción de migrantes. Cabe señalar que en la región, las zonas rurales, con menos de 2500 habitantes, albergan al $26.37 \%$ de la población municipal. Las actividades productivas son comercio y servicios, $54.8 \%$; industria, $39.1 \%$, y sector primario, $5.3 \%$ (ONU Hábitat, 2016).

\section{Participantes}

Como se ha señalado, las prácticas de crianza tienen un efecto en la personalidad, las elecciones futuras y el desarrollo personal y profesional de los/as adolescentes (Niedorys 
et al., 2018), por lo que es pertinente estudiar el fenómeno en este grupo etario. Dado que la investigación es un insumo para la orientación del desarrollo de política pública, a través del Observatorio Ciudadano de Seguridad del Municipio de San Juan del Río, Querétaro, se buscó concretar un estudio con representatividad municipal, de modo que se diseñó una muestra aleatoria y representativa de estudiantes de bachillerato de este municipio, que incluyó dos estratos (rural y urbano) y conglomerados (grupos en las escuelas). En cada estrato, la selección de escuelas se hizo de modo independiente, utilizando el método de Probabilidad Proporcional al Tamaño (PPT).

Se partió del padrón de escuelas registradas en la Secretaría de Educación Pública (SEP), donde consta la existencia de 32 escuelas en San Juan del Río, con una población de 11018 alumnos, distribuidos en 22 escuelas urbanas, con 6401 alumnos, y 10 rurales, con 4617 alumnos. De esta población, se calculó una muestra con un nivel de confianza del $95 \%, \mathrm{p}=0.9$, y un margen de error del $1.5 \%$-considerando el consumo de alcohol en jóvenes queretanos (Meza de Luna \& Concepción Valverde, 2016), principal variable de interés de un estudio más amplio sobre conductas de riesgo, del que deriva el presente escrito-, resultando en una muestra de 1349 estudiantes.

Se invitó a participar a 28 escuelas, de las que 10 eran rurales, pero sólo 5 aceptaron tomar parte en el estudio, por lo que finalmente participaron 23 escuelas (que en total albergaban a 6010 alumnos); 18 urbanas (con 4839 alumnos) y 5 rurales (con 1 171 alumnos).

Para las escuelas en muestra, se hizo una lista de los grupos de estudiantes que la conformaban. Se tomó con igual probabilidad una muestra de dos grupos de cada escuela $\mathrm{y}$, al interior de cada uno, se aplicó el cuestionario a todos los estudiantes que asistieron a clase el día de la aplicación. Sin embargo, se descartó el $4.85 \%$ de los 1714 cuestionarios aplicados, debido a que los estudiantes no quisieron contestar o sus respuestas no cumplieron con características de confiabilidad, de modo que se hicieron ajustes a los pesos de la muestra, que finalmente estuvo compuesta por las respuestas validadas de un total de 1630 participantes (50.39\% mujeres, Tabla 1$)$ de entre 14 y 21 años $(\mu=15.87$ años, ES =0.15, [IC: 15.57 a 16.17]).

De acuerdo al diseño muestral implementado, se utilizó el módulo de encuestas complejas del paquete estadístico sPSS en el análisis de datos. 
Tabla 1.

Población estudiantil de las escuelas seleccionadas en la muestra y número de participantes por escuela San Juan del Río, Querétaro, diciembre de 2016

\begin{tabular}{ccc}
\hline Escuela & Total de alumnos por escuela & Participantes \\
\hline Urbana & & \\
1 & 1718 & $16 *^{*}$ \\
2 & 769 & 81 \\
3 & 428 & 71 \\
4 & 427 & 85 \\
5 & 359 & 54 \\
6 & 238 & $15 *^{*}$ \\
7 & 237 & 71 \\
8 & 132 & 109 \\
9 & 103 & 48 \\
10 & 89 & 87 \\
11 & 82 & 61 \\
12 & 74 & 57 \\
13 & 54 & 53 \\
14 & 53 & 53 \\
15 & 45 & 22 \\
16 & 31 & 31 \\
\hline Rural & & 69 \\
1 & 336 & 64 \\
2 & 320 & 101 \\
3 & 316 & 113 \\
4 & 119 & 80 \\
5 & 80 &
\end{tabular}

*Escuelas de doble turno (matutino/vespertino), independientes en el padrón de la SEP. Muestra: dos grupos por turno. Fuente: Elaboración propia.

Las personas con quienes vivían los/as adolescentes eran, en orden decreciente, la madre (92.89\%), el padre (82.00\%), hermanas/os (84.80\%), abuelas/os (15.61\%), amistades $(4.17 \%)$, conocidos $(3.05 \%)$, padrastro $(2.30 \%)$ y madrastra $(1.23 \%)$. En cuanto a su situación económica, $4.56 \%$ de los/as adolescentes se identificaron con una situación mala; $40.73 \%$, regular; $47.53 \%$, buena; y $7.17 \%$, muy buena.

Cabe resaltar que se buscaba estudiar las prácticas de crianza de al menos una persona adulta que fungiera como padre o madre; para ello, se consideró en el análisis que los/as adolescentes vivieran con padre y madre, o alguno de los dos.

\section{Instrumento}

Se utilizó el Cuestionario a Estudiantes (Chávez et al., 2010) probado y avalado por el Instituto Nacional de Psiquiatría "Ramón de la Fuente" y la SEP, precisamente para ser 
aplicado a alumnos de secundaria y preparatoria. Se retomaron los apartados del cuestionario asociados con datos censales y relaciones familiares.

Las relaciones familiares se analizaron a partir de un instrumento de 16 preguntas sobre la interacción de adolescentes con sus padres y madres o sustitutos/as. El cuestionario tiene una alta confiabilidad $(\alpha=0.96)$ y opciones de respuesta con escala Likert de cinco puntos (de nunca a casi siempre). Cinco de los 16 ítems son concordantes con el modelo de democratización familiar $(\alpha=0.82)$, que pone en el centro una comunicación positiva en la que se sustentan discusiones abiertas y directas enmarcadas de sentimientos de afecto mutuo (Esteinou, 2008). También, incluye decisiones en las que se toma en cuenta la opinión de los/as hijos/as, apoyo emocional y límites claros. Por ejemplo, prácticas como involucrarse en los intereses de los/as adolescentes, conversar sobre sus amistades, incluirles para planear actividades, felicitarles por sus logros y expresarles afecto.

Otros cinco ítems son congruentes con prácticas de negligencia/permisividad $(\alpha=$ 0.90), donde existe un bajo perfil en el control o cuidado en la crianza (Sengrin \& Flora, 2011), tales como tener inconsistencia en los castigos (e. g., no cumplirlos o levantarlos antes de tiempo), dejar salir sin acordar hora de regreso o sin conocer con cuáles amistades sale, o que los padres/madres olviden en dónde estaban sus hijos/as. Finalmente, los otros seis ítems se relacionan con el autoritarismo, que llega a devenir en violencia $(\alpha=0.92)$; por ejemplo, un contexto familiar con maltrato físico o emocional, discusiones, mala relación con el padre o la madre o haber padecido abuso sexual.

\section{Procedimiento}

Se partió del padrón de escuelas de nivel medio superior registradas ante la SEP. En cada estrato, se hizo una selección aleatoria de escuelas y grupos. Se contactó a los directores de las escuelas seleccionadas para explicarles el objetivo, el procedimiento de la investigación, e invitarles a participar. Se programaron las aplicaciones en los grupos aleatoriamente seleccionados. La aplicación del instrumento tuvo una duración de entre 35 y 45 minutos, propia de una investigación más amplia para medir conductas de riesgo. El personal encargado de esta tarea fue capacitado para aplicar el cuestionario.

El protocolo se apegó a las pautas éticas estipuladas por la American Psychological Association (APA, 2005). Para ello, se realizó un consentimiento informado por escrito con los padres, las madres o tutores, y un asentimiento verbal con los/as adolescentes. En todo momento, se respetaron la confidencialidad y el anonimato de las personas, que 
participaron voluntariamente. El protocolo de investigación fue avalado por el Comité de Ética de la Universidad Autónoma de Querétaro.

\section{Análisis de los datos}

Para realizar el estudio comparativo en función del género y las zonas rural y urbana, se aplicaron, con un intervalo de confianza (IC) del $95 \%$, pruebas de independencia chicuadrada de Pearson, y estudio por razón de momios (RM) para el análisis de factores de riesgo, en función del estrato y del género. Todas las variables de las relaciones familiares se ajustaron a respuestas dicotómicas $\left(\mathrm{Sí}_{1}^{=}\right.$alta frecuencia / No = baja frecuencia) para realizar el último análisis.

\section{RESULTADOS Y DISCUSIÓN}

Participaron 1630 estudiantes de bachillerato (58.10\% del área urbana y $41.90 \%$ de la rural): la mayoría de los/as adolescentes refiere experimentar prácticas de crianza positiva (Figura 1), lo cual hace ver que en la cultura hay una tendencia a cambiar la lógica de crianza tradicional basada en el autoritarismo. Sin embargo, más de la tercera parte de los/as adolescentes señala tener padres/madres con rasgos permisivos, que tienden a relajar la disciplina. Las tres prácticas preponderantes que se alejan de la crianza positiva, reportadas por los/as adolescentes, son (Figura 1): 1. Te permiten salir con amigos/as que ellos no conocen $(40.47 \%) ; 2$. Cuando haces algo mal, tus papás amenazan con castigarte pero no lo cumplen (36.37\%); y 3. Te levantan los castigos antes de tiempo (33.69\%).

Alrededor de la cuarta parte de los/as adolescentes dice experimentar prácticas de crianza negligentes en las que no se les brinda apoyo emocional y/o les falta comunicación con sus padres/madres. Asimismo, se encuentran expuestos/as a la violencia: más de una cuarta parte vive en un ambiente de discusiones y, aunque en mucha menor medida, también da cuenta de violencia verbal, maltrato físico y abuso sexual en el hogar (Figura 1). A la luz de otros estudios en población mexicana, estos hallazgos podrían representar un problema en lo inmediato y a largo plazo, porque existe una asociación entre las prácticas de crianza negativas con mayores problemas conductuales y emocionales de los/as adolescentes (Méndez Sánchez et al., 2019). Además, el maltrato a los/as adolescentes se asocia con niveles más altos de reactividad emocional y con el uso habitual de respuestas no adaptativas a la angustia, incluidas la rumia y las conductas impulsivas 
(Heleniak, Jenness, Vander Stoep, McCauley \& McLaughlin, 2016), y el consumo de sustancias psicoactivas (Dubowitz et al., 2016).

También se encontró una mayor tendencia a malas relaciones con el padre que con la madre, de casi el doble (Figura 1). Siguiendo los argumentos de Disiye \& Mulambula (2015), podría deberse a que las madres tienden a ser las que más se involucran en el cuidado. De hecho, estos mandatos de género en el ejercicio de la crianza también se han encontrado en otros estudios con adolescentes en México, donde se sigue confirmando que las madres mantienen mayor comunicación, cuidado y cercanía con sus hijos/as que los padres (Barcelata \& Gutiérrez, 2018; Eguiarte \& Arenas, 2018; Méndez Sánchez et al., 2019). Estas condicionantes de género, aún presentes, constituyen una tarea pendiente para enfocar esfuerzos que promuevan prácticas de crianza positivas con los hombres, que abonen al desarrollo de los/as adolescentes y de las relaciones familiares más placenteras, en tanto que se sabe que la buena comunicación y las relaciones sanas del padre con sus hijos/as adolescentes, fortalecen el bienestar con atributos como la felicidad y la satisfacción de vivir, y reaniman la confianza que les permite una vida más plena (Disiye \& Mulambula, 2015). 


\section{Figura 1.}

Comparativo de la percepción de estudiantes de bachillerato

sobre las prácticas de crianza en la muestra total y por estrato rural y urbano

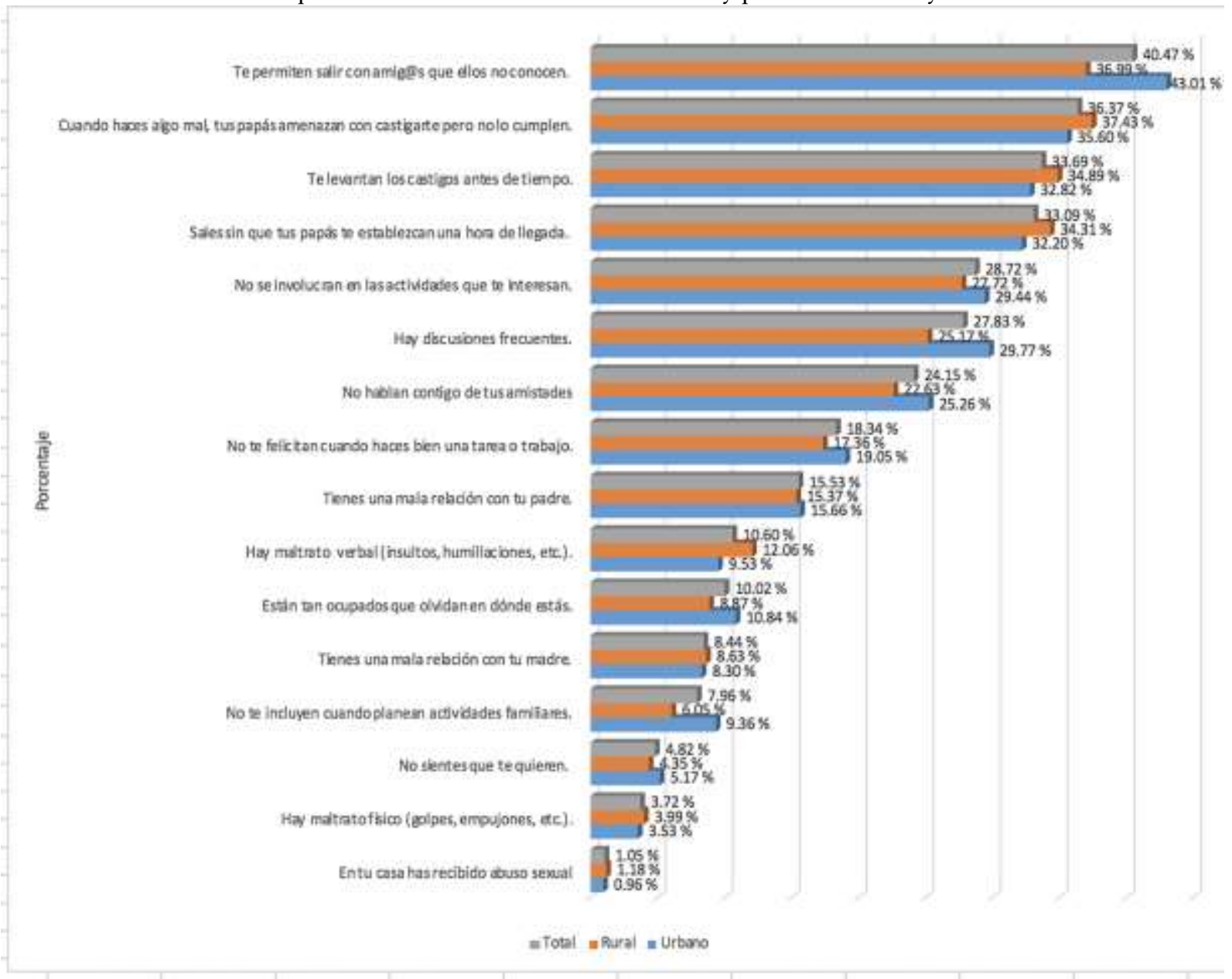

Fuente: Elaboración propia.

Al hacer el análisis por género, se observó una percepción de prácticas de crianza más laxas con los hombres que con las mujeres. Específicamente, con respecto a las mujeres, poco menos del doble de los hombres, dice que les permiten salir con amistades que sus padres/madres no conocen y sin establecerles hora de llegada (Tabla 2). Tales prácticas corroboran otros estudios que señalan que existe una socialización diferenciada entre hijas e hijos (Brown, Craig, \& Halberstadt, 2015) que tienden a perpetuar los estereotipos y las inequidades de género (Leaper \& Farkas, 2015), siendo esta diferencia en el trato que ejercen padres/madres un factor que coadyuva a modelar las identidades en la adolescencia (Sánchez, Whittaker, Hamilton \& Arango, 2017). 
Tabla 2.

Frecuencia y factores de riesgo en las prácticas de crianza asociadas con el género. Estudiantes de bachillerato San Juan del Río, Querétaro, diciembre de 2016

\begin{tabular}{|c|c|c|c|c|}
\hline $\begin{array}{l}\text { Característica en las } \\
\text { relaciones familiares }\end{array}$ & $\begin{array}{c}\text { Total } \\
\mathrm{N}=1630 \\
\%(\mathrm{IC} \text { a } 95 \%)\end{array}$ & $\begin{array}{c}\text { Mujeres } \\
858(50.39 \%) \\
\%(\text { IC a } 95 \%)\end{array}$ & $\begin{array}{c}\text { Hombres } \\
772(49.61 \%) \\
\%(\text { IC a } 95 \%)\end{array}$ & $\begin{array}{l}\mathrm{RM}(\mathrm{IC} \text { a } 95 \%) \\
\chi^{2}\end{array}$ \\
\hline $\begin{array}{l}\text { No te felicitan cuando haces bien } \\
\text { una tarea o un trabajo. }\end{array}$ & $18.34(16.42$ a 20.43$)$ & $18.37(15.91$ a 21.12$)$ & $18.30(14.85$ a 22.34$)$ & $\begin{array}{c}1.00(0.71 \text { a } 1.39), \\
0.00\end{array}$ \\
\hline No sientes que te quieren. & $4.82(3.34$ a 6.93$)$ & $4.75(2.94$ a 7.59$)$ & $4.90(3.35$ a 7.12$)$ & $\begin{array}{c}1.03(0.65 \text { a } 1.66), \\
0.00\end{array}$ \\
\hline $\begin{array}{l}\text { No se involucran en las } \\
\text { actividades que te interesan. }\end{array}$ & $28.72(23.97$ a 33.98$)$ & $28.95(23.97$ a 34.49$)$ & $28.48(23.38$ a 34.19$)$ & $\begin{array}{c}0.98(0.82 \text { a } 1.17) \\
0.04\end{array}$ \\
\hline $\begin{array}{l}\text { No hablan contigo de tus } \\
\text { amistades. }\end{array}$ & 24.15 (20.85 a 27.79) & $23.02(18.34$ a 28.47$)$ & $25.31(22.00$ a 28.94$)$ & $\begin{array}{c}1.13(0.84 \text { a } 1.53), \\
1.14\end{array}$ \\
\hline $\begin{array}{l}\text { No te incluyen cuando planean } \\
\text { actividades familiares. }\end{array}$ & $7.96(6.46$ a 9.76$)$ & 9.77(7.02 a 13.43) & $6.06(4.23$ a 8.60$)$ & $\begin{array}{c}0.60(0.33 \text { a } 1.09) \\
7.45\end{array}$ \\
\hline $\begin{array}{l}\text { Cuando haces algo mal, amenazan } \\
\text { con castigarte pero no lo cumplen. }\end{array}$ & $36.37(31.79$ a 41.21) & $38.16(32.20$ a 44.51) & $34.52(29.12$ a 40.36$)$ & $\begin{array}{c}0.85(0.62 \text { a } 1.17) \\
2.29\end{array}$ \\
\hline $\begin{array}{l}\text { Te levantan los castigos antes de } \\
\text { tiempo. }\end{array}$ & 33.69 (30.72 a 36.79) & $33.79(30.33$ a 37.44$)$ & $33.59(28.30$ a 39.92$)$ & $\begin{array}{c}0.99(0.72 \text { a } 1.36) \\
0.01\end{array}$ \\
\hline $\begin{array}{l}\text { Están tan ocupados que olvidan en } \\
\text { dónde estás. }\end{array}$ & $10.02(8.59$ a 11.65$)$ & 10.50(7.91 a 13.82) & 9.51(7.53 a 11.96) & $\begin{array}{c}0.90(0.56 \text { a } 1.42) \\
0.44\end{array}$ \\
\hline $\begin{array}{l}\text { Sales sin que te establezcan una } \\
\text { hora de llegada. }\end{array}$ & $33.09(29.68$ a 36.69$)$ & $23.28(20.27$ a 26.59$)$ & $43.18(38.03$ a 48.48$)$ & $\begin{array}{c}2.50(1.92 \text { a 3.27), } \\
71.51 * *\end{array}$ \\
\hline $\begin{array}{l}\text { Te permiten salir con amigos/as } \\
\text { que ellos no conocen. }\end{array}$ & $40.47(35.40$ a 45.75$)$ & $28.91(25.23$ a 32.89$)$ & $52.41(44.88$ a 59.84$)$ & $\begin{array}{c}2.71(2.06 \text { a } 3.56) \\
91.77 * *\end{array}$ \\
\hline $\begin{array}{l}\text { Tienes una mala relación con tu } \\
\text { padre. }\end{array}$ & $15.53(13.55$ a 17.75$)$ & $16.16(13.57$ a 19.31$)$ & $14.90(11.91$ a 18.49$)$ & $\begin{array}{c}0.91(0.65 \text { a } 1.27) \\
0.48\end{array}$ \\
\hline $\begin{array}{l}\text { Tienes una mala relación con tu } \\
\text { madre o su sustituta. }\end{array}$ & $8.44(7.26$ a 9.80$)$ & $8.57(6.43$ a 11.34$)$ & $8.31(6.27$ a 10.93$)$ & $\begin{array}{c}0.97(0.57 \text { a } 1.63) \\
0.03\end{array}$ \\
\hline $\begin{array}{l}\text { Hay maltrato físico (golpes, } \\
\text { empujones, etc.). }\end{array}$ & $3.72(2.52$ a 5.47$)$ & $3.28(1.86$ a 5.74$)$ & $4.18(2.59$ a 6.67$)$ & $\begin{array}{c}1.28(0.63 \text { a } 2.62), \\
0.90\end{array}$ \\
\hline $\begin{array}{l}\text { Hay maltrato verbal (insultos, } \\
\text { humillaciones, etc.). }\end{array}$ & $10.60(8.08$ a 13 a 78$)$ & $11.09(7.56$ a 15.99$)$ & $10.09(7.12$ a 14.10$)$ & $\begin{array}{c}0.90(0.52 \text { a } 1.54) \\
0.43\end{array}$ \\
\hline Hay discusiones frecuentes. & $27.83(23.50$ a 32.61$)$ & $30.92(24.38$ a 38.32$)$ & $24.65(21.02$ a 28.67$)$ & $\begin{array}{c}0.73(0.53 \text { a } 1.01) \\
7.83\end{array}$ \\
\hline $\begin{array}{l}\text { En tu casa has recibido abuso } \\
\text { sexual. }\end{array}$ & $1.05(0.41$ a 2.66$)$ & & $2.13(0.84$ a 5.29$)$ & \\
\hline
\end{tabular}

Cabe destacar que en este estudio no se encontraron diferencias estadísticas en la percepción de las prácticas de crianza violentas en función del género (Tabla 2), lo que contradice otros estudios que muestran que a los hombres, más que a las mujeres, se les llega a aplicar medidas disciplinarias (Calvete, Guadix \& Orue, 2010) de tipo violento, como castigos físicos y verbales (González \& Hernández, 2012; Arrebola, Gijón, Garzón \& Gómez, 2019), lo cual se ha explicado, en el contexto mexicano, como producto de los estereotipos de género existentes en nuestra cultura, donde se justifica en mayor medida un trato más enérgico y con poca comunicación hacia los hijos (varones), mientras que a las hijas se les trata con mayor protección, cuidado y con una comunicación más abierta que auspicia la expresión de emociones (González \& Hernández, 2012). Sin embargo, la literatura no muestra una 
evidencia conclusiva al respecto, en tanto que también se ha encontrado lo opuesto en madres yucatecas (Flores, Morales, Cortés, Campos \& Díaz Loving, 2019), por lo que aún falta desarrollar más investigación que permita dilucidar estas contradicciones.

Por otra parte, sorprende el hallazgo de $2.13 \%$ de abuso sexual en hombres (Tabla 2), mientras que ninguna mujer señaló haberlo padecido, contraviniendo la evidencia de otras investigaciones, donde se asocia una mayor frecuencia de esta violencia hacia las mujeres que hacia los hombres (Scoglio, Kraus, Saczynski, Jooma, \& Molnar, 2019). La razón para esta diferencia podría residir en que el cuestionario hace una prospección general de "haber recibido abuso sexual" en el último año, lo cual dista de ser una batería amplia para indagar conductas específicas asociadas al abuso sexual (i.e., sin contacto, con contacto; y dentro de éste, con penetración). Además, siendo que la indagatoria se constriñe a las experiencias recientes del último año, se descartan los eventos anteriores, lo cual es una limitante en tanto que el abuso sexual ocurre más frecuentemente en la adolescencia temprana (Scoglio et al., 2019), sobrepasada por la edad promedio de los/as participantes de este estudio. También habría que considerar el tabú asociado al hecho, ya que puede limitar su reporte. Estos aspectos pudieran contribuir a presentar un sub-registro en el indicador, por lo que futuras investigaciones podrían hacer un aporte al incluir cuestionarios específicos.

Al considerar sólo el contexto, no se encontró diferencia estadísticamente significativa en ninguna de las prácticas de crianza entre estudiantes del medio urbano y rural $\left(\chi^{2}<6\right.$ en todos los casos, $\left.\mathrm{gl}=1, \mathrm{P}>0.05\right)$. Este hallazgo está en concordancia con otros estudios con adolescentes, mostrando una tendencia reciente a la homogeneidad entre los contextos rurales y urbanos (Kafetsios \& Karaolanis, 2016), por lo menos en lo referente a algunos aspectos de salud mental (Thorsteinsson, Ryan \& Sveinbjornsdottir, 2013) y psicológica (Lariscy, Reber \& Paek, 2011). Esta incipiente tendencia a la homogenización entre ambos contextos pudiera encontrar su explicación en la influencia de la globalización, que, entre otras cosas, apareja el uso de la tecnología y de la Internet, y llega a repercutir en la identidad de los/as adolescentes, en su cultura y tradiciones, lo cual se refleja, a su vez, en los modelos de crianza, que se ejercen cada vez más uniformemente (Huntsinger, Shaboyan \& Karapetyan, 2019). También hay que considerar que diversas investigaciones apuntan hacia un continum, y no tanto a la antigua dicotomía rural/urbano (Dixon \& Chartier, 2016), en parte por expansión de las manchas urbanas a las zonas rurales, el incremento y accesibilidad de los medios de transporte y la construcción de parques industriales en zonas rurales cercanas a vías de comunicación de primer orden (e.g., la carretera 57), dando lugar a corredores industriales, como el de Querétaro-San Juan, que a su vez integra a los municipios de Pedro Escobedo y El Marqués. Durante las décadas de 1990 y 2000, en estos tres 
municipios (excluyendo Querétaro) se concentraron $26 \%$ de micro empresas, $43 \%$ de pequeñas, $53 \%$ de medianas y $51 \%$ de las grandes empresas establecidas en el Estado. Para 2009, en la producción estatal bruta, San Juan del Río se ubicó en segundo lugar, con 19.4 \%, después de Querétaro que registró 56.8 \% (Censo Económico, INEGI).

De acuerdo con Arias (1992), la instalación industrial en áreas rurales provocó que se redujera drásticamente la población dedicada a la agricultura, a tal grado que en poco tiempo se consideró como una labor marginal, conformando una nueva estructura laboral. Esta transformación dio lugar a una importante disociación del lugar de residencia y de trabajo. En San Juan del Río, buena parte de la población rural se incorporó al mercado laboral urbanoindustrial desde los años 80, fenómeno que cobró aún más fuerza en la década siguiente, con la incorporación de las mujeres al mercado de trabajo.

El proceso de urbanización implica cambios espaciales, pero también socioculturales; estos últimos se pueden analizar desde el modo de vida - entendido como modelo cultural(Remy \& Voyé, 1992). De manera que la modernización de San Juan del Río -asociada indiscutiblemente con la industrialización- produjo cambios no sólo territoriales, sino sociales y culturales de profundo alcance, que impactaron las relaciones familiares (Nieto, 2000), lo que explica, en parte, que en el presente estudio no se observen distinciones sustantivas entre lo rural y lo urbano como antaño, sino una tendencia un tanto más homogeneizadora de ambos contextos, es decir, a una clara urbanización del modo de vida.

Sin embargo, al considerar las prácticas de crianza negligentes, permisivas y violentas, en función tanto del género como del contexto (Tabla 3), se evidencia que existe mayor riesgo para los hombres ACU que los ACR de que sus padres/madres: 1. No hablen con ellos acerca de sus amistades (RM = 0.65 [IC: 0.43 a 0.97], urbana $28.85 \%$ vs. rural $20.80 \%) ; 2$. No los incluyan cuando planean actividades familiares (RM $=0.41$ [IC: 0.19 a 0.89], urbana $8.08 \%$ vs. rural $3.51 \%)$; y 3. Que perciban más discusiones al interior de sus familias $(\mathrm{RM}=0.56$ [IC: 0.36 a 0.85], urbana $29.30 \%$ vs. rural $18.75 \%)$. No se encontraron diferencias estadísticamente significativas entre las mujeres de los dos contextos. Estos hallazgos, en cuanto a las relaciones menos cercanas con sus padres/madres en los adolescentes (varones) de áreas urbanas, con respecto a las rurales, son similares a los que describen Zhang y Fuligni (2006). Al tiempo, esta comparativa por género y contexto permite visibilizar un campo inexplorado en México y abona a la poca literatura en este sentido. 
Tabla 3. Frecuencia y factores de riesgo en las prácticas de crianza asociadas con el género en áreas rurales y urbanas. Estudiantes de bachillerato. San Juan del Río, Querétaro, diciembre del 2016.

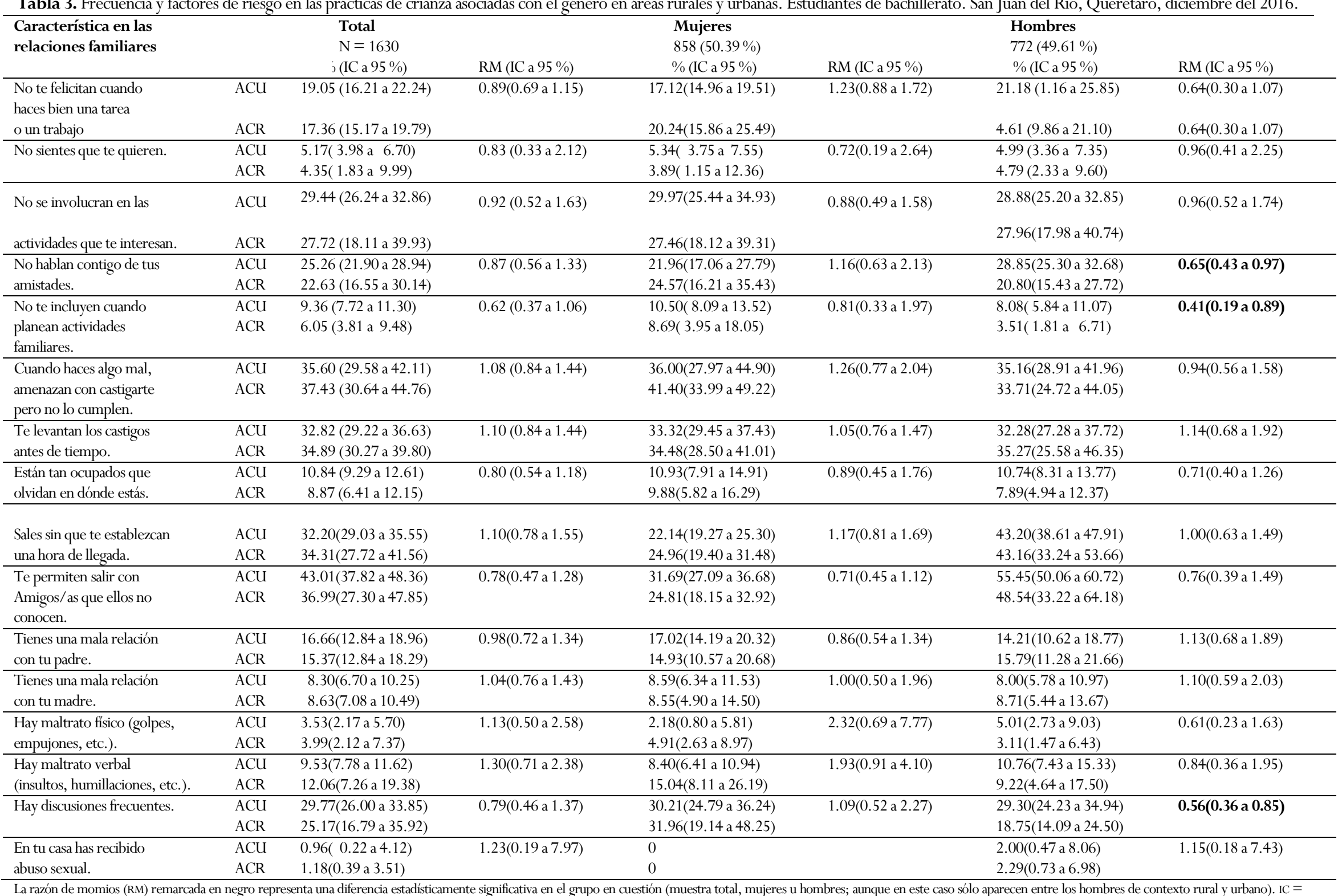

\begin{tabular}{lcc} 
abuso sexual. $\quad$ ACR & $1.18(0.39$ a 3.51$)$ & 0 \\
\hline La razón de momios (RM) remarcada en negro representa una diferencia estadísticamente significativa en el grupo en cuestión (muestra total, mujeres u hombres; aunque en este caso sólo aparecen entre los hom & $2.29(0.73$ a 6.98$)$
\end{tabular}

intervalo de confianza; $\mathrm{ACU}=$ adolescentes de contextos urbanos; $\mathrm{ACR}=$ adolescentes de contextos rurales. Fuente: Elaboración propia. 
Por último, llama la atención que los hombres ACR sean quienes reportan que en sus casas hay menos discusiones, siendo esta diferencia significativa con todos los demás adolescentes (Tabla 3). Estos indicadores apuntan hacia los hombres ACR, que al parecer perciben menos la violencia, lo cual deberá estudiarse a profundidad en futuras investigaciones. Sin embargo, y a manera de indicio, estas percepciones de las "discusiones" pudieran estar entrelazadas con, al menos, dos de los aportes de los estudios sobre la violencia: 1. La normalización de la violencia con la que pudieran estar evaluando los hombres ACR (Meza de Luna, Westendarp Palacios, Palacios Sierra \& Cantera Espinoza, 2015), y 2. Dado que a las mujeres se les habla más de la violencia que a los hombres, como una estrategia preventiva (Oster, 2018), ellas tienen una mayor habilidad para percibirla (Wang, 2019), lo que podría hacer a las mujeres ACR más proclives a detectarla.

En suma, los hallazgos del presente estudio permiten elucidar las percepciones de los/as adolescentes para ser tomadas en cuenta en el desarrollo de intervenciones. Además, ponen de manifiesto que aún queda una brecha para alcanzar prácticas de crianza positiva. Al respecto, Capano Bosh, González Tornaría, Navarrete y Mels (2018) mencionan que es menester el desarrollo de programas de educación de madres/padres para prevenir la violencia y fortalecer su función de crianza no sólo en la infancia, sino también en la adolescencia, cuando se tiende a relajar el cuidado y acompañamiento.

Los resultados de este estudio deberán interpretarse considerando las siguientes limitantes: 1. Se basó en una muestra representativa del municipio de San Juan del Río, Querétaro, por lo que sus resultados pudieran no ser generalizables para otras poblaciones. 2. Está ausente la perspectiva de padres/madres, por lo que futuras investigaciones harían un aporte al dimensionar esto, y contrastarlo con lo que reportan sus hijos/as, así como explorar los significados que se le atribuyen a las prácticas de crianza. 3. La investigación consultó las prácticas de crianza que padres y madres ejercen en conjunto, por lo que en próximas indagatorias sería deseable diferenciar las conductas de cada uno/a, de manera que permitan hacer un análisis que evalúe los sesgos en función del género de quien ejerce y recibe dichas prácticas. 4. El estudio no contempla otras variables mediadoras, como la condición de migración o la estructura familiar (e. g., si se es hijo/a primogénito/a, único/a o si se tiene uno/a o más hermanos/as mayores), que pudieran influir en las prácticas de crianza.

\section{CONCLUSIONES}

En este estudio se han presentado indicadores de las percepciones de adolescentes sobre las prácticas de crianza de sus padres/madres en una muestra representativa del municipio de San 
Juan del Río, Querétaro, dejando al descubierto algunas condiciones que entrañan la vida de uno de los sectores más vulnerables de la población: la adolescencia.

Encontramos una tendencia de las familias hacia prácticas de crianza democráticas, lo que plantea un panorama esperanzador. Parecería que la disciplina autoritaria tradicional está cediendo paso a alternativas diferentes. Sin embargo, encontramos que prevalece un alto grado de permisividad (40\%). En menor medida (25\%), aún persiste el trato violento $\mathrm{y}$ negligente a los adolescentes. Así, este estudio ha dimensionado el nivel de prácticas de crianza no-positivas (i.e., permisivas, negligentes y violentas) que pudiera poner en riesgo el desarrollo integral de los jóvenes, y ha aportado conocimiento actualizado sobre el tema, a fin de atajar su invisibilidad y fortalecer su prevención.

Se encontró que los/as adolescentes perciben que existe un sesgo de género en el trato que reciben. La disciplina llega a ser más laxa con los hombres que con las mujeres, evidenciando que aún persiste una socialización diferenciada entre hijas e hijos, lo que contribuye a perpetuar los estereotipos y las inequidades de género, en tanto que el ejercicio de la voluntad y del poder se distribuye inequitativamente. Esto podría deberse, ciertamente, a que en México se vive una violencia exacerbada hacia las mujeres y ello podría aparejar un ánimo proteccionista. Sin embargo, eso no quita el resultado: la construcción social y psicológica de las diferencias entre los géneros, de modo que sigue pendiente la articulación social para proveer espacios más seguros para todas/os las/os adolescentes.

Por otro lado, al analizar las prácticas de violencia que reciben los/as adolescentes en función del género, no encontramos diferencia significativa. Sin embargo, al hacer un análisis más fino de las prácticas de crianza incluyendo el contexto (rural/urbano) y el género, hallamos que existe mayor riesgo para los adolescentes varones de áreas urbanas, que de las rurales, de ser más ignorados y de vivir en un ambiente familiar de discusiones, mientras que no se encontraron diferencias significativas entre las mujeres de los dos contextos (rural/urbano). Estos hallazgos ponen de manifiesto que el trato hacia los/as adolecentes está mediado por el género y el contexto urbano/rural, de ahí la pertinencia de hacer estudios comparativos considerando el contexto. Dada la escasez de información comparativa sobre las prácticas de crianza entre los contextos urbano y rural, este estudio hace un aporte en ese sentido.

Encontramos otro sesgo de género en las relaciones entre los/as adolescentes con sus progenitores/as, siendo que establecen mejores relaciones con las madres que con los padres, lo cual hace patente la necesidad de fortalecer programas de masculinidades que favorezcan las relaciones de los hombres-padres con sus hijos/as. Finalmente, aunque las diferencias de las relaciones en la familia entre los géneros y los contextos parecen estar acortándose, quedan 
aquí evidenciadas algunas prácticas que perpetúan la operación de reglas familiares diferenciadas en función del género y del contexto, demostrando las brechas vigentes para alcanzar los estadios de crianza positiva que permitan a los/as adolescentes construir futuros más prometedores, con menor riesgo y vulnerabilidad.

\section{REFERENCIAS}

Andrade, P., Betancourt, D., Vallejo, A., Segura, B. \& Rojas, R. M. (2012). Prácticas parentales y sintomatología depresiva en adolescentes. Salud mental, 35(1), pp. 29-36.

American Psychological Association/apa (2005). Publication manual of the American Psychological Association (5a ed.). Washington, DC: American Psychological Association.

ARDELT, M. \& ECCLES, J. (2001). Effects of mothers 'Parental efficacy beliefs and promotive parenting strategies on inner-city youth. Journal of Family Issues. 22(8), pp. 944-972.

Arias, P. (1992). Nueva rusticidad mexicana. México: CONACULTA.

Arrebola, I. A., Gijón, G. G., Garzón, F. R. \& Gómez, M. D. M. O. (2019). La percepción de los adolescentes de las prácticas parentales desde la perspectiva de género. Pedagogía social: revista interuniversitaria, 33, pp. 125-136.

Barcelata, E. \& Gutiérrez, V. (2018). Percepción de prácticas parentales en adolescentes tempranos y tardíos: una perspectiva del desarrollo. Investigación y Práctica en Psicología del Desarrollo, 4, pp. 1-14.

Betancourt, D., González, A., Cota, A. F., García, D., Hernández, K. \& Salazar, M. (2018). Prácticas parentales y consumo de marihuana en universitarios. Psicología Iberoamericana, 26(1), pp. 31-41.

Brown, G. L., Craig, A. B. \& Halberstadt, A. G. (2015). Parent gender differences in emotion socialization behaviors vary by ethnicity and child gender. Parenting, 15(3), pp. $135-157$.

Calvete, E., Guadix, M. G. \& Orue, I. (2010). El Inventario de Dimensiones de Disciplina (DDI), Versión niños y adolescentes: Estudio de las prácticas de disciplina parental desde una perspectiva de género. Anales de Psicología/Annals of Psychology, 26(2), pp. 410-418.

Cantón, D., Ramírez, M. A. \& Cantón, J. (2014). Papel moderador del sexo en las prácticas de crianza. Revista INFAD de Psicología "International Journal of Developmental and Educational Psychology", 1(1), 275-284. 
Capano Bosh, A., González Tornaría, M. L., Navarrete, I. \& Mels, C. (2018). Del castigo físico a la parentalidad positiva. Revisión de programas de apoyo parental. Revista de Psicología, 14(27), pp. 125-138.

Casais, D., Flores, M. \& Domínguez, A. (2017). Percepción de prácticas de crianza: análisis confirmatorio de una escala para adolescentes. Acta de Investigación Psicológica, 7(2), pp. 2717-2726.

Charalampous, K., Demetriou, C., Tricha, L. \& Loannou, M. (2018). The effect of parental style on bullying and cyber bullying behaviors and the mediating role of peer attachment relationships : A longitudinal study. Journal of Adolescence, 64, pp. 109-123.

Chávez, J., Villatoro, J., Robles, L., Bretón, M., SÁnchez, V. \& Gutiérrez, M. (2010). Encuesta escolar sobre adicciones en el Estado de Jalisco. México: Instituto Nacional de Psiquiatría Ramón de la Fuente Muñiz. Recuperado de https: / / bit.ly/3lxEF0m.

Consejo de Europa, Comité de Ministros (2006). Recomendación Rec (2006) 19 del Comité de Ministros a los Estados Miembros sobre políticas de apoyo al ejercicio positivo de la parentalidad. Recuperado de https: / / bit.ly/3twNdY5.

Cruz, Y., Flores, L. M. \& JimÉneZ, J. (2019). Prácticas parentales y ansiedad como predictoras del consumo de alcohol en adolescentes. Enseñanza e Investigación en Psicología, pp. 55-64.

Dangi, T. \& WiTt, P. A. (2016). Parenting styles and positive youth development. Youth Development Initiative. Recuperado de https://bit.ly/3lETEFV.

Disiye, M. A. \& Mulambula, S. M. (2015). Adolescent emotional well-being: A comparative study of mother-and father-adolescent communication influence. American International Journal of Science, 4(6), pp. 96-104.

Dixon, M. A. \& Chartier, K. G. (2016). Use patterns among urban and rural residents: demographic and social influences. Alcohol Research, 38(1), pp. 69-77.

Dubowitz, H., Thompson, R., Arria, A. M., English, D., Metzger, R. \& Kotch, J. B. (2016). Characteristics of child maltreatment and adolescent marijuana use: A prospective study. Child Maltreatment, 21(1), pp. 16-25.

Eguiarte, B. E. B. \& Arenas, V. G. (2018). Percepción de prácticas parentales en adolescentes tempranos y tardíos: Una perspectiva del desarrollo. Investigación y Práctica en Psicología del Desarrollo, 4, pp. 1-14.

Eldad, R. \& BenATOv, J. (2008). Adult attachment and perceived parental style may shape leadership behaviors. Leadership and Organization Development Journal, 39(2), pp. 261 275. 
Eraso, J., Bravo, Y. \& Delgado, M. (2006). Creencias, actitudes y prácticas sobre crianza en madres cabeza de familia en Popayán: un estudio cualitativo. Revista de Pediatría, 41(3), pp. 23-40.

Esteinou, R. (2008). La familia nuclear en México: lecturas de su modernidad. Siglos XVI al XX. México: CIESAS y Porrúa.

Estrada, J. J. \& RodrígueZ, B. (2012). Prácticas parentales, autoestima, inicio de la relación sexual y uso de sustancias adictivas en estudiantes de nivel medio superior de Chiapas, México. Revista Internacional de Estudios en Educación, 12(2), pp. 102-117.

Flores, M. M., Morales, M. T., Cortés, M. D. L., Campos, M. J. C. \& Díaz loving, R. (2019). Crianza materna y regulación emocional en adolescentes: diferencias asociadas al sexo. Emerging Trends in Education, 1(2), pp. 96-120.

Giddens, A. \& Sutton, P. (2014). Sociología (Séptima edición). Madrid: Alianza Editorial.

GONZÁLEZ, J. \& HERNÁNDEZ, A. (2012). Las prácticas educativas parentales: análisis por género de una muestra de adolescentes Coahuilenses. PSICUMEX, 2(2), pp. 42-53.

Heleniak, C., Jenness, J. L., Vander Stoep, A., McCauley, E. \& Mclaughlin, K. A. (2016). Childhood maltreatment exposure and disruptions in emotion regulation: A transdiagnostic pathway to adolescent internalizing and externalizing psychopathology. Cognitive Therapy and Research, 40(3), pp. 394-415.

Huntsinger, C. S., Shaboyan, T. \& Karapetyan, A. M. (2019). The influence of globalization on adolescents' conceptions of self and future self in rural and urban Armenia. New Directions for Child and Adolescent Development, 164, pp. 67-82.

IZZEDIN, R. \& PACHAJOA, A. (2009). Pautas, prácticas y creencias acerca de la crianza... ayer y hoy. Liberabit, 15(2), pp. 109-115.

INEGI (2015). Población rural y urbana. Cuéntame INEGI. Recuperado de https: / / bit.ly/3vOSI6b.

KAFETSIOS, K. \& KARAOLANiS, S. (2016). On social status, cultural orientation and well-being: A comparison between urban and rural areas in Greece. Recuperado de https: / / bit.ly/3cYqKfE.

LARISCY, R., ReBER, B. \& PAEK, H. J. (2011). Exploration of health concerns and the role of social media information among rural and urban adolescents: A preliminary study. International Electronic Journal of Health Education, 14, pp. 16-36.

LEAPER, C. \& FARKAS, T. (2015). The socialization of gender during childhood and adolescence. In GruSEC, J. E. \& HASTINGS, P. D. (Eds.), Handbook of socialization: Theory and research (pp. 541-565). New York, USA: Guilford Press. 
MÉndez SÁnchez, M., Peñaloza Gómez, R., García Méndez, M., Jaenes Sánchez, J. C. \& VelázQuez JuRAdo, H. R. (2019). Divergencias en la percepción de prácticas parentales, comportamiento positivo y problemáticas entre padres e hijos. Acta Colombiana de Psicología, 22(2), pp. 194-217.

Meza de luna, M. E. \& Concepción Valverde, P. (2016). Encuesta a la juventud. Consumo de sustancias psicoactivas en el Municipio de Querétaro 2015. México: Observatorio Ciudadano de Seguridad del Municipio de Querétaro.

, Westendarp-Palacios, P., Palacios-Sierra, P. \& Cantera-Espinoza, L. M. (2015). Never to me! Concealment of intimate partner violence in Querétaro, Mexico. Trames, 19(2), pp. 155-169.

Niedorys, B., Mikos, M., RzącA, M. \& KockA, K. (2018). Relationships in the family and their impact on the degree of the internet addiction among school youth. Journal of Education, Health and Sport, 8(4), pp. 346-356.

NiETO, J. (2000). Del hacendado al empresario. Querétaro. México: UAQ Y CUMDES.

Oman, R. F., Vesely, S. K., Tolma, E., Aspy, C. B., Rodine, S. \& Marshall, L. (2007). Does family structure matter in the relationships between youth assets and youth alcohol, drug and tobacco use? Journal of Research on Adolescence, 17(4), pp. 743-766.

ONU HÁBitat (2018). Índice básico de las ciudades prósperas. San Juan del Río. México: ONU Habitat, INFONAVIT. Recuperado de https:/ / bit.ly/3lDAuQK.

Oster, M. (2018). Preventing sexual violence starts with what we teach our boys. The Education Digest, No. 83(6), pp. 18-20.

PARDINI, D. \& LOEBER, R. (2008). Interpersonal callousness trajectories across adolescence: Early social influences and adult outcomes. Criminal Justice and Behavior, 35(2), pp. 173 196.

REMY, J. \& VOYÉ, L. (1992.). La ville: vers une novvelle definition. Paris: Editions L'Harmattan.

Rengifo, A. L. M. \& MARTíneZ, L. O. (2014). Discursos y prácticas de crianza en la primera infancia: una construcción sociocultural de las relaciones de género y generación en la familia. Revista Tendencias y Retos, 19(2), 63-76.

Rodríguez Villamizar, L. A. \& Amaya-Castellanos, C. (2019). Estilos de crianza, autoeficacia parental y problemas conductuales infantiles en tres municipios de Santander. Rev Univ Ind Santander Salud, 51(3), pp. 228-238.

Ruvalcaba Romero, N. A., Gallegos-Guajardo, J., Caballo, V. E. \& Villegas Guinea, D. (2016). Prácticas parentales e indicadores de salud mental en adolescentes. Psicología desde el Caribe, 33(3), pp. 223-236. 
Sánchez, D., Whittaker, T. A., Hamilton, E. \& Arango, S. (2017). Familial ethnic socialization, gender role attitudes, and ethnic identity development in Mexican-origin early adolescents. Cultural Diversity and Ethnic Minority Psychology, 23(3), pp. 335-347.

Scoglio, A. A., Kraus, S. W., Saczynski, J., Jooma, S. \& Molnar, B. E. (2019). Systematic review of risk and protective factors for revictimization after child sexual abuse. Trauma, Violence, and Abuse, pp. 1-20.

SEgrin, C. \& FlorA, J. (2011). Family communication (Second edition). New York: Routledge. Smetana, J. G. (2017). Current research on parenting styles, dimensions, and beliefs. Current opinion in psychology, 15, pp. 19-25.

SNYDER, J. (2016). Coercive family processes and the development of child social behavior. In DiSHION, T. \& SNYDER, J. (Eds.), The Oxford handbook of coercive relationship dynamics (pp. 101-113). Nueva York: Oxford University Press.

Thorsteinsson, E. B., Ryan, S. \& Sveinbjornsdottir, S. (2013). The mediating effects of social support and coping on the stress-depression relationship in rural and urban adolescents. Open Journal of Depression, 2(1), pp. 1-6.

Valente, J. Y., Cogo, H. \& Zila, M. (2018). Predicting latent classes of drug use among adolescents through parental alcohol use and parental style: a longitudinal study. Social Psychiatry and Psychiatric Epidemiology, 54(4), pp. 455-467.

VArgas Rubilar, J. \& ArÁn FiliPPETTI, V . (2014). Importancia de la crianza para el desarrollo cognitivo infantil: una revisión teórica. Revista Latinoamericana de Ciencias Sociales, Niñez y Juventud, 12(1), pp. 171-186.

Voth, J. (2001). Relationships between rural/urban living, family environment, and coping strategies. Recuperado de https://bit.ly/3sjtYkJ.

Waller, R., Gardner, F. \& Hyde, L. W. (2013). What are the associations between parenting, callous-unemotional traits, and antisocial behavior in youth? A systematic review of evidence. Clinical Psychology Review, 33(4), pp. 593-608.

WANG, L. (2019). Education, perception factors, and prevention of intimate partner violence: Empirical research on chinese university students' perceptions and attitudes concerning intimate partner violence. Journal of Interpersonal Violence, 34(8), pp. 1611-1632.

Yu, J. J., Wang, Y. J., Xu, J. J., Li, M., Chen, X., Ding, N. \& Wu, F. (2010). Relationships between family-related factors and middle students' test anxiety in the urban and rural areas of Harbin. Chinese Journal of Child Health Care, 4(18).

ZHANG, W. \& FULIGNi, A. J. (2006). Authority, autonomy, and family relationships among adolescents in urban and rural China. Journal of Research on Adolescence, 16(4), pp. 527537. 